\title{
THE INFRARED COLORS OF THE SUN
}

\author{
L. Casagrande ${ }^{1}$, I. Ramírez ${ }^{2}$, J. Meléndez $^{3}$, And M. Asplund ${ }^{1}$ \\ ${ }^{1}$ Research School of Astronomy and Astrophysics, Mount Stromlo Observatory, The Australian National University, ACT 2611, Australia; luca@mso.anu.edu.au \\ ${ }^{2}$ McDonald Observatory and Department of Astronomy, University of Texas at Austin, 1 University Station, C1400 Austin, TX 78712-0259, USA \\ ${ }^{3}$ Departamento de Astronomia do IAG/USP, Universidade de São Paulo, Rua do Mãtao 1226, São Paulo, 05508-900 SP, Brazil \\ Received 2012 August 31; accepted 2012 September 26; published 2012 November 20
}

\begin{abstract}
Solar infrared colors provide powerful constraints on the stellar effective temperature scale, but they must be measured with both accuracy and precision in order to do so. We fulfill this requirement by using line-depth ratios to derive in a model-independent way the infrared colors of the Sun, and we use the latter to test the zero point of the Casagrande et al. effective temperature scale, confirming its accuracy. Solar colors in the widely used Two Micron All Sky Survey (2MASS) $J H K_{s}$ and WISE $W 1-4$ systems are provided: $(V-J)_{\odot}=1.198,(V-H)_{\odot}=1.484$, $\left(V-K_{s}\right)_{\odot}=1.560,(J-H)_{\odot}=0.286,\left(J-K_{s}\right)_{\odot}=0.362,\left(H-K_{s}\right)_{\odot}=0.076,(V-W 1)_{\odot}=1.608$ $(V-W 2)_{\odot}=1.563,(V-W 3)_{\odot}=1.552$, and $(V-W 4)_{\odot}=1.604$. A cross-check of the effective temperatures derived implementing 2MASS or WISE magnitudes in the infrared flux method confirms that the absolute calibration of the two systems agrees within the errors, possibly suggesting a $1 \%$ offset between the two, thus validating extant near- and mid-infrared absolute calibrations. While 2MASS magnitudes are usually well suited to derive $T_{\text {eff }}$, we find that a number of bright, solar-like stars exhibit anomalous WISE colors. In most cases, this effect is spurious and can be attributed to lower-quality measurements, although for a couple of objects ( $3 \% \pm 2 \%$ of the total sample) it might be real, and may hint at the presence of warm/hot debris disks.
\end{abstract}

Key words: stars: fundamental parameters - Sun: fundamental parameters - techniques: photometric

Online-only material: color figures, machine-readable table

\section{INTRODUCTION}

Photometric systems and filters carry information on various fundamental stellar properties, such as effective temperature $\left(T_{\text {eff }}\right)$, metallicity $([\mathrm{Fe} / \mathrm{H}])$, and surface gravity $(\log g)$. Also, when studying more complex systems, integrated magnitudes and the colors of stars can be used to infer properties of the underlying stellar populations, by interpreting observations via theoretical population synthesis models. However, stars with well-known physical parameters and colors are needed to establish how observed photometric data must be translated into physical quantities and placed on an absolute scale. The absolute calibration of photometric systems precisely deals with this matter, and it has a long and noble history, especially in using solar-type stars for this purpose (e.g., Johnson 1965; Wamsteker 1981; Campins et al. 1985; Rieke et al. 2008). Arguably, the star with the best-known parameters, as well as the most important benchmark in astrophysics, is the Sun, but for obvious reasons it cannot be observed with the same instruments and under the same conditions applied to distant stars, thus making it virtually impossible to directly measure its colors (Stebbins \& Kron 1957).

Photometry of stars with stellar properties very similar to the Sun provides a way to cope with this limit, although it is not obvious how to identify stars satisfying such a condition in the first place. Linking photometric measurements to stellar parameters is in fact the goal, and selecting Sun-like stars based on colors would clearly introduce a circular argument. On the other hand, spectroscopy provides an excellent way of determining $T_{\text {eff }}, \log g$, and $[\mathrm{Fe} / \mathrm{H}]$ in stars, and it is routinely used for this purpose, although it can be heavily model dependent. Nevertheless, this major limit is easily overcome by restricting our investigation to a purely differential analysis of stars with spectra largely identical to a reference one. If the latter is solar, then it is thus possible to identify the stars most closely resembling the Sun, the so-called solar twins. ${ }^{4}$

Over the last few years, some of us (Meléndez et al. 2006, 2009; Meléndez \& Ramírez 2007; Ramírez et al. 2009) have conducted a systematic search aimed at characterizing and discovering the best solar twins in the local $\sim 100 \mathrm{pc}$ volume, starting from an initial sample of about 100 stars in the Hipparcos catalog chosen to be broadly consistent with being solar-like. For each candidate, high-resolution, high signal-to-noise ratio observations were conducted and compared to solar reference spectra (which in fact are reflected sunlight of asteroids) obtained with the same instrumentation and within each observing run (at McDonald and Las Campanas observatories; see Section 2 in Ramírez et al. 2012).

Because the procedure adopted to identify solar twins does not assume any a priori $T_{\text {eff }}$, solar twins have already been used to set the zero point of the effective temperature scale via the infrared flux method (IRFM; Casagrande et al. 2010). Therefore, the effective temperature scale is a basic factor in measuring metallicities and, by comparison with theoretical isochrones, for deriving stellar ages. Thus, the zero point of the effective temperature scale directly impacts basic constraints of Galactic chemical evolution models (e.g., the metallicity distribution function and the age-metallicity relation), and it is important to correctly interpret the Sun in a Galactic context (e.g., Nordström et al. 2004; Casagrande et al. 2011; Datson et al. 2012). Because of its far-reaching implications, we have continued to investigate this topic (see also Huber et al. 2012, for a comparison between the angular diameters measured by interferometry and those obtained via the IRFM); in particular, we have conducted dedicated observations to

\footnotetext{
4 According to their increasing similarity to the Sun, stars can be classified as solar-like, solar analogs, and solar twins (Cayrel de Strobel 1996). The term "solar twin" does not imply that the stars were born together with the Sun.
} 
overcome the major bottleneck in linking stellar parameters to photometry, i.e., the availability of homogeneous and highaccuracy photometric data. In Meléndez et al. (2010), we have presented new Strömgren $u v b y$ observations of more than 70 solar analogs and have derived the colors of the Sun in this system, which then have been used to investigate the zero point of various metallicity scales. Similarly, in Ramírez et al. (2012), we have presented new $U B V(R I)_{\mathrm{C}}$ photometry of 80 solar analogs and derived solar colors in the widely used Johnson-Cousins system, obtaining a definitive value for the long-debated value of $(B-V)_{\odot}=0.653 \pm 0.003$.

In this paper, we finally focus on the infrared colors of the Sun and the tight constraints they can provide on the $T_{\text {eff }}$ scale. In fact, even though it is possible to use Strömgren and Johnson-Cousins colors (Meléndez et al. 2010; Ramírez et al. 2012), infrared colors are better suited to this purpose, being nearly independent of blanketing and surface gravity effects for the spectral types considered here (e.g., Bessell et al. 1998).

In addition to this motivation, highly standardized and precise infrared photometry is available from all-sky surveys nowadays, essentially defining new standard systems for the years to come: Two Micron All Sky Survey (2MASS) in the near-infrared and the WISE satellite in the mid-infrared. Accurate solar colors in these two systems are thus crucial for a number of purposes. Most importantly, the reliability at which infrared measurements can be used to infer stellar properties must also be assessed: while 2MASS data can be confidently adopted in most cases, a number of stars seem to exhibit anomalous WISE colors. We find that most of those are artifacts that can be avoided by imposing more stringent observational constraints, although in a few cases they might be real and indicate the presence of debris disks.

\section{SAMPLE AND PHOTOMETRIC DATA}

Our sample consists of the 112 stars used in Ramírez et al. (2012), from whom we have adopted $V$ magnitudes and stellar parameters. The latter were derived using excitation and ionization equilibrium conditions (Ramírez et al. 2012 and references therein), and because of the strictly differential analysis with respect to the solar reference spectrum, the impact of systematic errors is minimized across the limited parameter space covered by our sample. Although a few stars in our sample might have faint companions, the effect seems negligible for 2MASS, but we detected a few anomalous stars in the WISE colors. As we discuss later, these stars were discarded from the analysis.

For each star, we have queried the 2MASS $J(1.25 \mu \mathrm{m})$, $H(1.65 \mu \mathrm{m}), K_{s}(2.17 \mu \mathrm{m})$ and the WISE W1 $(3.4 \mu \mathrm{m}), W 2$ $(4.6 \mu \mathrm{m}), W 3(12 \mu \mathrm{m}), W 4(22 \mu \mathrm{m})$ catalogs (Cutri et al. 2003, 2012 , respectively) for photometry. Some of the bright targets have saturated or unreliable 2MASS magnitudes; to retain the best data, in a given band we consider only observations with photometric quality flag "A," read flag "1," blend flag " 1 " (i.e., one component fit to the source), and contamination and confusion flag " 0 " (i.e., source unaffected by known artifacts). ${ }^{5}$ This set of flags automatically retains stars with photometric uncertainty (msigcom) in a given band better than $0.06 \mathrm{mag}$, and for the full sample mean errors are $0.02 \mathrm{mag}$ in $J$ and $K_{s}$ and 0.03 mag in the $H$ band.

Similarly, for WISE observations, we restrict our analysis to measurements consistent with being point sources (ext $=0$, meaning that no band has a reduced $\chi^{2}>3$ and the source

\footnotetext{
See http://www.ipac.caltech.edu/2mass/releases/allsky/doc/sec2_2a.html.
}

is not within 5 arcsec of a 2MASS Extended Source Catalogue entry), unaffected by known artifacts (ccf $=0)$, quality flag "A" and variability flag "n," or 0-5 (i.e., most likely not variable). ${ }^{6}$ The "A" quality flag implies a signal-to-noise ratio higher than 10, automatically curbing large photometric uncertainties. As detailed in the WISE Explanatory Supplement, ${ }^{7}$ the $W 1-4$ channels saturate at $\sim 8.1,6.7,3.8$, and $-0.4 \mathrm{mag}$, respectively, although fits to the unsaturated wings of the pointspread function allow viable magnitudes to be obtained up to $2.0,1.5,-3.0$, and $-4.0 \mathrm{mag}$. Given the brightness of our targets, this is never a concern as the saturated pixel fraction is on average 0.07 in $W 1$ and essentially drops to zero in the other bands. Finally, to further decrease the possibility of having spurious identifications, we also require each WISE source to have a $2 \mathrm{MASS}$ point-source counterpart associated with it. All WISE sources identified with the above constraints have a position offset smaller than 3 arcsec (average 1.5) with respect to the target coordinate. Some $20 \%-30 \%$ of the stars do fall severely apart from the main locus of the color- $T_{\text {eff }}$ relations, especially in the $W 2$ and $W 4$ bands (no such effect is visible using 2MASS), although all quality records listed above are fulfilled (Figure 1); somewhat arbitrarily, we exclude stars having $V-W 2 \geqslant 1.65$ and $V-W 4 \geqslant 1.80$, and we briefly discuss this in Section 4. Altogether, this set of choices limits the mean (max) error to $0.03(0.05), 0.02$ (0.02), 0.02 (0.02), and 0.07 $(0.11)$ in $W 1, W 2, W 3$, and $W 4$, respectively.

\section{THE SOLAR INFRARED COLORS}

For solar-type stars, $V-J, V-H$, and $V-K_{s}$ are known to display a remarkably tight correlation with $T_{\text {eff }}$, while being nearly independent of other parameters such as $[\mathrm{Fe} / \mathrm{H}]$ and $\log g$. The strong temperature sensitivity in solar-type stars is due to the long wavelength baseline, which almost brackets the region of maximum flux in these stars, covering part of the spectrum with similar continuum opacity but differing temperature sensitivity to the Planck function; this argument continues to hold also when replacing 2MASS with WISE filters (Figure 1).

In the literature, there are various calibrations relating optical/near-infrared indices to effective temperatures of giants and dwarfs (e.g., Ridgway et al. 1980; Alonso et al. 1996; Ramírez \& Meléndez 2005; Casagrande et al. 2010; Boyajian et al. 2012a); for the set of filters used in this work, it can easily be estimated that a change of about 0.03 mag in solar colors implies an uncertainty of about $50 \mathrm{~K}$ on the zero point of the $T_{\text {eff }}$ scale. Therefore, to check the reliability of various $T_{\text {eff }}$ scales, accurate and precise colors must be derived. This is done model independently in Section 3.1, while in Section 3.2 we check on the zero point of our effective temperature scale.

For this reason, it is important that the colors under investigation are obtained directly, without resorting to conversions between different systems. Cousins (1987a, 1987b) provides relations between the Strömgren and Johnson-Cousins photometry, and more recently, Bilir et al. $(2008,2011)$ have derived an extensive set of color transformations relating the 2MASS, WISE, and $B V(R I)_{\mathrm{C}}$ systems. Using those, the solar colors derived in Meléndez et al. (2010), Ramírez et al. (2012), and here are usually reproduced within 0.05 mag (with better performances when transforming from the Strömgren system), although certain color combinations are offset by as much as $0.10 \mathrm{mag}$, which

\footnotetext{
6 http://wise2.ipac.caltech.edu/docs/release/allsky/expsup/sec2_2a.html 7 http://wise2.ipac.caltech.edu/docs/release/allsky/expsup/sec6_3d.html
} 

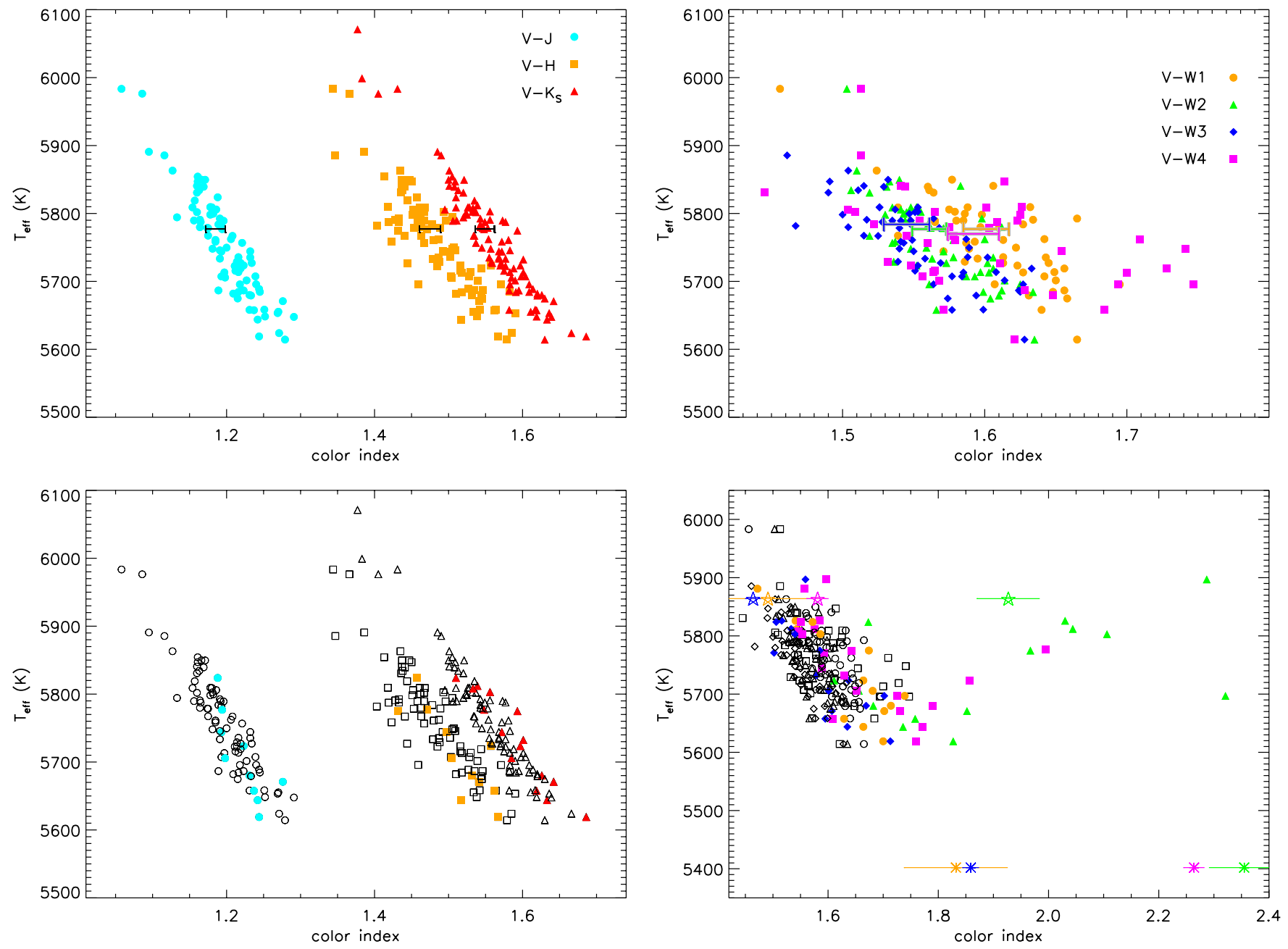

Figure 1. Color indices vs. effective temperatures obtained from the IRFM. Top panels: using only stars satisfying the photometric criteria discussed in Section 2. The

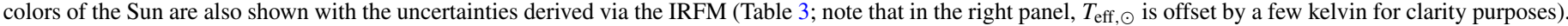
Bottom panels: objects having $V-W 2 \geqslant 1.65$ or $V-W 4 \geqslant 1.80$ but satisfying all other photometric and quality constraints of Section 2 are highlighted in colors. Stars from the upper panels are now shown with empty circles. In the bottom right panel, the effective temperature and color ranges have been increased to include HD 69830 (asterisk); also shown is HD 72905 (pentagram). For both objects, WISE's photometric error bars are shown (see further discussion in Section 4).

are nevertheless still consistent with the standard deviation of the transformations reported in Bilir et al. (2008, 2011).

\subsection{Spectral Line-depth Ratios}

Here, we use the line-depth ratio (LDR) technique as described in Ramírez et al. (2012) to derive the infrared colors of the Sun in a model-independent way. Briefly, this technique exploits the fact that the ratios of depths of spectral line pairs with very different excitation potential are excellent $T_{\text {eff }}$ indicators - thus correlating well with observed colors-essentially independent of $[\mathrm{Fe} / \mathrm{H}]$ and $\log g$ (e.g., Gray 1994). For mainsequence stars with $v \sin i \lesssim 5 \mathrm{~km} \mathrm{~s}^{-1}$, as is the case for our stars, LDRs are also weakly dependent on rotational broadening (Biazzo et al. 2007). For each set of line pairs (from Kovtyukh et al. 2003), we measured the ratios in all of the stars of our sample and linearly fitted those ratios as a function of the color index under consideration (Figure 2), after the exclusion of stars not satisfying the photometric quality requirements discussed in Section 2. From each fit, the standard deviation of the fit minus the data residual $\left(\sigma_{\mathrm{fit}}\right)$ was also obtained. Note that only line pairs for which the color versus LDR slope was greater than 0.3 have been used. Slopes shallower than this imply a lower sensitivity, leading to larger errors in the derived solar color. Since the slope
Table 1

$\left(V-K_{s}\right)_{\odot}$ Color Inferred from LDR Measurements

\begin{tabular}{lccccccc}
\hline \hline $\begin{array}{l}\lambda_{1} \\
(\AA)\end{array}$ & Species & $\begin{array}{c}\lambda_{2} \\
(\AA)\end{array}$ & Species & $N_{\star}$ & $\sigma_{\text {fit }}$ & $\left(V-K_{s}\right)_{\odot}$ & $\sigma_{\text {ss }}$ \\
\hline 5490.15 & Ti I & 5517.53 & Si I & 85 & 0.038 & 1.555 & 0.011 \\
5645.62 & Si I & 5670.85 & V I $_{\text {I }}$ & 85 & 0.036 & 1.556 & 0.014 \\
5650.71 & Fe I & 5670.85 & V I & 85 & 0.036 & 1.558 & 0.009 \\
5665.56 & Si I & 5670.85 & V I $_{\text {I }}$ & 85 & 0.036 & 1.558 & 0.016 \\
5665.56 & Si I & 5703.59 & V I & 85 & 0.031 & 1.567 & 0.016 \\
\hline
\end{tabular}

Notes. $N_{\star}$ is the number of stars used for the fit, $\sigma_{\text {fit }}$ is the standard deviation of the fit minus data residual, and $\sigma_{\mathrm{ss}}$ is the standard deviation of the color inferred from the nine reflected sunlight asteroid observations used for solar reference.

(This table is available in its entirety in a machine-readable form in the online journal. A portion is shown here for guidance regarding its form and content.)

errors are about 0.03 , this criterion is equivalent to a $10 \sigma$ cut. An example of all line pairs used to derive the solar $\left(V-K_{s}\right)$ color is given in Table 1. The interpolation of those fits at the solar ratio (measured in the reflected sunlight of asteroids with the same procedure used for stars) returns the color index of the Sun. Since we have nine reflected sunlight observations, nine solar LDR values are available for each line pair, resulting in 


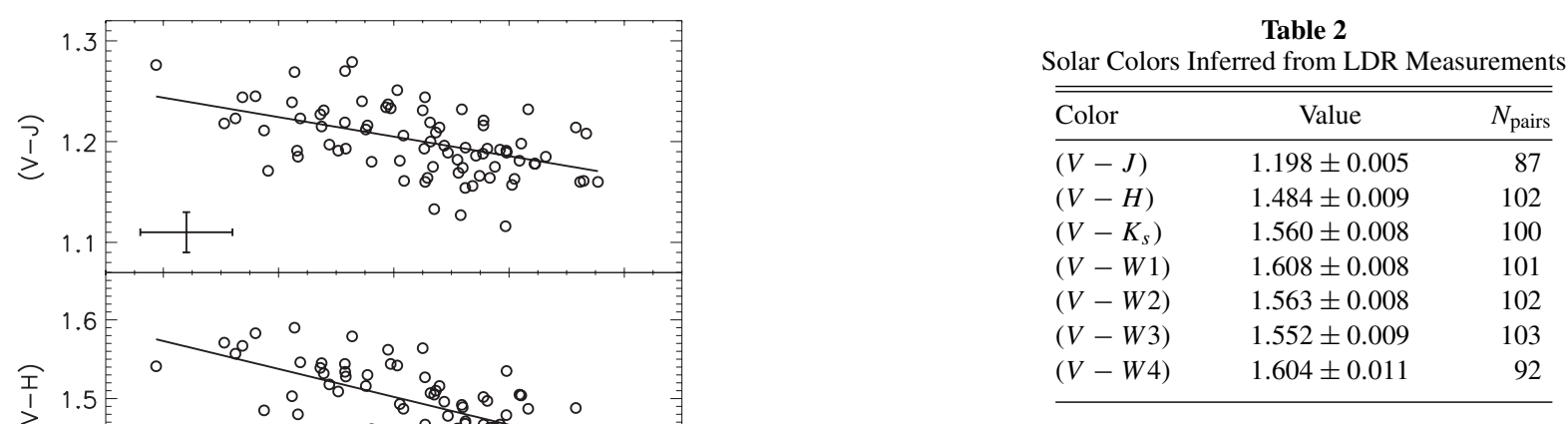

Table 3

Solar Colors Inferred from Different $T_{\text {eff }}$ Measurements: Spectroscopic (i.e., Excitation and Ionization Equilibrium) and the IRFM Implemented Using Tycho2 + 2MASS (TY2M) or Johnson-Cousins + 2MASS (JC2M) Photometry

\begin{tabular}{lcccr}
\hline \hline Color & Spectroscopic & IRFM $_{\text {TY2M }}$ & IRFM $_{\text {JC2M }}$ & $N_{\text {stars }}$ \\
\hline$(V-J)$ & $1.207 \pm 0.013$ & $1.197 \pm 0.013$ & $1.185 \pm 0.013$ & 87 \\
$(V-H)$ & $1.499 \pm 0.014$ & $1.489 \pm 0.013$ & $1.475 \pm 0.014$ & 87 \\
$\left(V-K_{s}\right)$ & $1.572 \pm 0.013$ & $1.563 \pm 0.013$ & $1.549 \pm 0.013$ & 95 \\
$(V-W 1)$ & $1.620 \pm 0.015$ & $1.613 \pm 0.014$ & $1.601 \pm 0.016$ & 52 \\
$(V-W 2)$ & $1.576 \pm 0.011$ & $1.570 \pm 0.011$ & $1.561 \pm 0.012$ & 56 \\
$(V-W 3)$ & $1.564 \pm 0.013$ & $1.558 \pm 0.014$ & $1.545 \pm 0.016$ & 59 \\
$(V-W 4)$ & $1.610 \pm 0.021$ & $1.601 \pm 0.018$ & $1.592 \pm 0.018$ & 42 \\
\hline
\end{tabular}

of the stellar parameters (e.g., $\left.T_{\text {eff }}, \log g,[\mathrm{Fe} / \mathrm{H}]\right)$ relevant to a given color index and derive that of the Sun by solving with respect to its parameters (e.g., Holmberg et al. 2006; Meléndez et al. 2010; Ramírez et al. 2012). Works focusing on the $T_{\text {eff }}$ scale adopt essentially the same approach, where the colors of the Sun are inferred by reverting polynomial color $-[\mathrm{Fe} / \mathrm{H}]-T_{\text {eff }}$ relations derived for dwarf stars (e.g., Ramírez \& Meléndez 2005; Casagrande et al. 2006). While indices in Table 2 tightly correlate with $T_{\text {eff }}$, because of the relatively narrow parameter space covered by our stars, we have verified that they do not display any significant dependence on $\log g$ or $[\mathrm{Fe} / \mathrm{H}]$. In fact, performing a multiple linear regression with respect to all three parameters or $T_{\text {eff }}$ only did not improve on the residual, or change it within $0.001 \mathrm{mag}$ the values derived. Such a simple linear relation also has the advantage of making the connection between a given color index and the underlying $T_{\text {eff }}$ scale straightforward. Depending on the band considered, several tens of stars survive the quality cuts we impose on 2MASS/WISE photometry (Section 2 and Table 3). Using the spectroscopic (excitation and ionization equilibrium) temperatures determined for the full sample of solar analogs returns solar indices systematically redder by $\sim 0.01$ mag with respect to those obtained via LDRs. This implies that on average our spectroscopic $T_{\text {eff }}$ are overestimated by about $20 \mathrm{~K}$, in agreement with what is found by Ramírez et al. (2012) using optical indices.

For all stars in our sample, we also run the IRFM to derive effective temperatures uncorrelated to the spectroscopic analysis. In fact, the IRFM is essentially model independent and is affected very little by the metallicity and surface gravity of each star, the most relevant factor being the absolute calibration of the photometric systems adopted. Since all these stars are nearby, reddening is also not a concern, as confirmed by using intrinsic Strömgren color calibrations (Meléndez et al. 2010). In Casagrande et al. (2010), the zero point of the IRFM scale was calibrated using solar twins having Tycho2 and 2MASS photometry. Now, the availability of $U B V(R I)_{\mathrm{C}}$ magnitudes 
allows us to check whether this is also the case when using instead the Johnson-Cousins system. ${ }^{8}$ Replacing the Tycho 2 with the Johnson-Cousins system in the IRFM returns effective temperatures that are cooler by $26 \pm 4 \mathrm{~K}(\sigma=41 \mathrm{~K})$, which is within the zero-point uncertainty of our effective temperature scale. The same conclusion is obtained by restricting the analysis to solar twins only, ${ }^{9}$ for which we obtain a median/mean $T_{\text {eff }}$ of $5787 / 5786 \mathrm{~K}$ and $5762 / 5750 \mathrm{~K}$ using Tycho 2 and Johnson-Cousins photometry, respectively. These differences are mirrored in the color indices of Table 3: those inferred using Johnson-Cousins in the IRFM are in fact slightly bluer than obtained via LDRs, by an amount that would be almost perfectly offset should the effective temperatures increase by $20 \mathrm{~K}$. As expected, $(V-J)_{\odot},(V-H)_{\odot}$, and $\left(V-K_{s}\right)_{\odot}$ derived here agree extremely well with those obtained by Casagrande et al. (2010), reverting color- $[\mathrm{Fe} / \mathrm{H}]-T_{\text {eff }}$ polynomials defined over a much wider parameter space. ${ }^{10}$

Effective temperatures determined using Tycho 2 photometry in the IRFM return color indices that agree almost perfectly with LDRs. These results confirm the overall good consistency obtained by implementing different photometric systems in the IRFM, with systematic uncertainties at the level of about $20 \mathrm{~K}$, i.e., $0.01 \mathrm{mag}$ in colors. Errors in Table 3 take this into account by adding such zero-point systematic uncertainties to the uncertainties derived analytically from the fits.

\section{THE WISE THROUGH EXCESS OF INFRARED IS MADE A FOOL?}

As discussed in Section 2, stars having exceedingly red indices in WISE (Table 4) were not used to derive solar colors, although all other photometric quality constraints were satisfied (apart from a few cases in a given band having ccf $\neq 0$ or a signal-to-noise ratio lower than 10 , bands that were always excluded from the analysis). The unusual colors of these stars are clearly visible in the bottom right panel of Figure 1 . The bands most strikingly affected are $W 2$ and partly $W 4$, while $W 1$ and $W 3$ seem only slightly offset to the red with respect to the main locus defined by the full sample. This sort of signature $(W 1-W 2 \gtrsim 0.3$ ) would not be entirely unexpected if looking at brown dwarfs. In fact, WISE's two shortest bands are designed to optimize sensitivity to this class of objects by probing their deep $\mathrm{CH}_{4}$ absorption band at $\sim 3.3 \mu \mathrm{m}(W 1)$ and the region relatively free of opacity at $\sim 4.6 \mu \mathrm{m}$ (W2), where their Planck function approximately peaks (e.g., Wright et al. 2010; Mainzer et al. 2011; Kirkpatrick et al. 2011).

To quantify the amount of contamination expected from a potential low-mass star companion, we combine a synthetic MARCS (Gustafsson et al. 2008) solar spectrum with that of a late-type $\mathrm{M}$ dwarf $\left(T_{\text {eff }}=2500 \mathrm{~K}\right)$ of the same metallicity, and assuming a secondary-to-primary radius ratio of 0.2 (Boyajian et al. 2012b), we conclude that the effect on the color indices shown in Figure 1 would be of the order of $0.01 \mathrm{mag}$. Therefore, even if the spectral features of a brown dwarf could account for the red $W 1-W 2$ index we observe, the overwhelming flux of the primary makes this solution not viable even for an $\mathrm{M}$ dwarf, in addition to the fact that it would still be difficult not to

\footnotetext{
For implementation of the IRFM using WISE magnitudes instead of 2MASS see the Appendix. Here, we prefer to use the 2MASS system because of the better-quality data.

9 That is, stars having spectroscopic stellar parameters within $1.4 \sigma$ from the solar ones, in accordance with the criterion used by Ramírez et al. (2012).

10 Incidentally, using optical and infrared solar colors from LDRs in the aforementioned polynomials returns an average $T_{\text {eff }}=5755 \pm 22 \mathrm{~K}$.
}

Table 4

Stars Having $V-W 2 \geqslant 1.65$ or $V-W 4 \geqslant 1.80$

\begin{tabular}{|c|c|c|c|c|c|}
\hline HIP & $V$ & $W 1$ & $W 2$ & $W 3$ & $W 4$ \\
\hline 8507 & 8.899 & $\begin{array}{l}7.185 \\
(1.45)\end{array}$ & $\begin{array}{l}7.217 \\
(1.25)\end{array}$ & $\begin{array}{l}7.230 \\
(1.00)\end{array}$ & $\begin{array}{l}7.109 \\
(1.09)\end{array}$ \\
\hline 11072 & 5.190 & $\begin{array}{l}3.607 \\
(0.28)\end{array}$ & $\begin{array}{l}2.903 \\
(2.09)\end{array}$ & $\begin{array}{l}3.631 \\
(2.12)\end{array}$ & $\begin{array}{l}3.594 \\
(1.04)\end{array}$ \\
\hline 12186 & 5.785 & $\begin{array}{l}4.241 \\
(0.73)\end{array}$ & $\begin{array}{l}3.741 \\
(2.25)\end{array}$ & $\begin{array}{l}4.252 \\
(1.71)\end{array}$ & $\begin{array}{l}4.210 \\
(0.87)\end{array}$ \\
\hline 15457 & 4.836 & $\begin{array}{l}3.334 \\
(0.38)\end{array}$ & $\begin{array}{l}2.614 \\
(1.21)\end{array}$ & $\begin{array}{l}3.334 \\
(1.66)\end{array}$ & $\begin{array}{l}3.243 \\
(0.96)\end{array}$ \\
\hline 22263 & 5.497 & $\begin{array}{l}3.956 \\
(0.37)\end{array}$ & $\begin{array}{l}3.467 \\
(2.04)\end{array}$ & $\begin{array}{l}3.981 \\
(1.37)\end{array}$ & $\begin{array}{l}3.912 \\
(0.93)\end{array}$ \\
\hline 29525 & 6.442 & $\begin{array}{l}4.768 \\
(0.33)\end{array}$ & $\begin{array}{l}4.475 \\
(1.70)\end{array}$ & $\begin{array}{l}4.857 \\
(0.76)\end{array}$ & $\begin{array}{l}4.800 \\
(0.91)\end{array}$ \\
\hline 38072 & 9.222 & $\begin{array}{l}7.627 \\
(1.57)\end{array}$ & $\begin{array}{l}7.678 \\
(0.90)\end{array}$ & $\begin{array}{l}7.626 \\
(1.26)\end{array}$ & $\begin{array}{l}7.227 \\
(1.21)\end{array}$ \\
\hline 38228 & 6.900 & $\begin{array}{l}5.198 \\
(0.42)\end{array}$ & $\begin{array}{l}5.048 \\
(1.30)\end{array}$ & $\begin{array}{l}5.293 \\
(0.79)\end{array}$ & $\begin{array}{l}5.170 \\
(1.17)\end{array}$ \\
\hline 44713 & 7.306 & $\begin{array}{l}5.650 \\
(0.49)\end{array}$ & $\begin{array}{l}5.629 \\
(1.26)\end{array}$ & $\begin{array}{l}5.744 \\
(1.14)\end{array}$ & $\begin{array}{l}5.717 \\
(0.96)\end{array}$ \\
\hline 55459 & 7.646 & $\begin{array}{l}5.965 \\
(0.63)\end{array}$ & $\begin{array}{l}5.990 \\
(1.53)\end{array}$ & $\begin{array}{l}6.045 \\
(0.92)\end{array}$ & $\begin{array}{l}5.994 \\
(0.89)\end{array}$ \\
\hline 57291 & 7.466 & $\begin{array}{l}5.802 \\
(1.05)\end{array}$ & $\begin{array}{l}5.730 \\
(1.25)\end{array}$ & $\begin{array}{l}5.831 \\
(1.35)\end{array}$ & $\begin{array}{l}5.694 \\
(1.16)\end{array}$ \\
\hline 66885 & 9.302 & $\begin{array}{l}7.638 \\
(1.05)\end{array}$ & $\begin{array}{l}7.691 \\
(0.88)\end{array}$ & $\begin{array}{l}7.665 \\
(1.13)\end{array}$ & $\begin{array}{l}7.445 \\
(0.92)\end{array}$ \\
\hline 77052 & 5.868 & $\begin{array}{l}4.129 \\
(0.65)\end{array}$ & $\begin{array}{l}3.547 \\
(2.02)\end{array}$ & $\begin{array}{l}4.166 \\
(1.73)\end{array}$ & $\begin{array}{l}4.143 \\
(0.83)\end{array}$ \\
\hline 80337 & 5.391 & $\begin{array}{l}3.919 \\
(0.74)\end{array}$ & $\begin{array}{l}3.378 \\
(2.03)\end{array}$ & $\begin{array}{l}3.902 \\
(0.65)\end{array}$ & $\begin{array}{l}3.834 \\
(0.74)\end{array}$ \\
\hline 85042 & 6.287 & $\begin{array}{l}4.676 \\
(0.89)\end{array}$ & $\begin{array}{l}4.240 \\
(2.14)\end{array}$ & $\begin{array}{l}4.708 \\
(0.85)\end{array}$ & $\begin{array}{l}4.657 \\
(0.82)\end{array}$ \\
\hline 88194 & 7.101 & $\begin{array}{l}5.472 \\
(0.94)\end{array}$ & $\begin{array}{l}5.343 \\
(1.32)\end{array}$ & $\begin{array}{l}5.506 \\
(1.74)\end{array}$ & $\begin{array}{l}5.492 \\
(1.15)\end{array}$ \\
\hline 96895 & 5.959 & $\begin{array}{l}4.385 \\
(0.47)\end{array}$ & $\begin{array}{l}4.004 \\
(1.66)\end{array}$ & $\begin{array}{l}4.444 \\
(0.78)\end{array}$ & $\begin{array}{l}4.412 \\
(0.92)\end{array}$ \\
\hline 100963 & 7.089 & $\begin{array}{l}5.517 \\
(0.52)\end{array}$ & $\begin{array}{l}5.416 \\
(1.08)\end{array}$ & $\begin{array}{l}5.583 \\
(0.75)\end{array}$ & $\begin{array}{l}5.538 \\
(1.06)\end{array}$ \\
\hline 109110 & 7.570 & $\begin{array}{l}5.870 \\
(1.22)\end{array}$ & $\begin{array}{l}5.743 \\
(1.01)\end{array}$ & $\begin{array}{l}5.857 \\
(1.18)\end{array}$ & $\begin{array}{l}5.810 \\
(0.92)\end{array}$ \\
\hline 113357 & 5.467 & $\begin{array}{l}3.881 \\
(0.29)\end{array}$ & $\begin{array}{l}3.361 \\
(1.75)\end{array}$ & $\begin{array}{l}3.927 \\
(2.38)\end{array}$ & $\begin{array}{l}3.914 \\
(0.84)\end{array}$ \\
\hline
\end{tabular}

Notes. Bands that do not satisfy all of the quality constraints discussed in Section 2 are written in italics. The $\chi^{2}$ of the source extraction in each band is given in parentheses.

affect $W 3$. Neither the alignment/confusion with extragalactic sources (which would be considerably fainter than our objects; see below) nor cool (sub)-stellar objects is likely. The angular resolution of WISE passes from 6.1 arcsec in $W 1$ to $12 \operatorname{arcsec}$ in $W 4$; using the higher resolution of 2MASS, none of the targets discussed here have more than one counterpart within 12 arcsec. All anomalous sources have $W 1$ brighter than $7.6 \mathrm{mag}$ (5.9 mag if considering the reddest $V-W 2>1.8)$. Using this constraint with the previous synthetic model $\left(T_{\text {eff }}=2500 \mathrm{~K}\right)$ would imply the presence of cool $\mathrm{M}$ dwarfs closer than 7.5 (3.5) pc and brighter than $V \sim 17$ (15.5) mag. Using instead a synthetic brown dwarf spectrum $\left(T_{\text {eff }}=1000 \mathrm{~K}\right.$; Burrows et al. 2006) and adopting $R=0.1 R_{\odot}$, the above estimates would change into distances closer than $1(0.5) \mathrm{pc}$ and $V$ magnitudes brighter than 21 (19.5) mag, thus making the superposition of brown dwarfs to our solar-like stars extremely unlikely.

Interpreting the color anomaly as mid-infrared emission could hint at the presence of warm/hot debris disks, even though this 

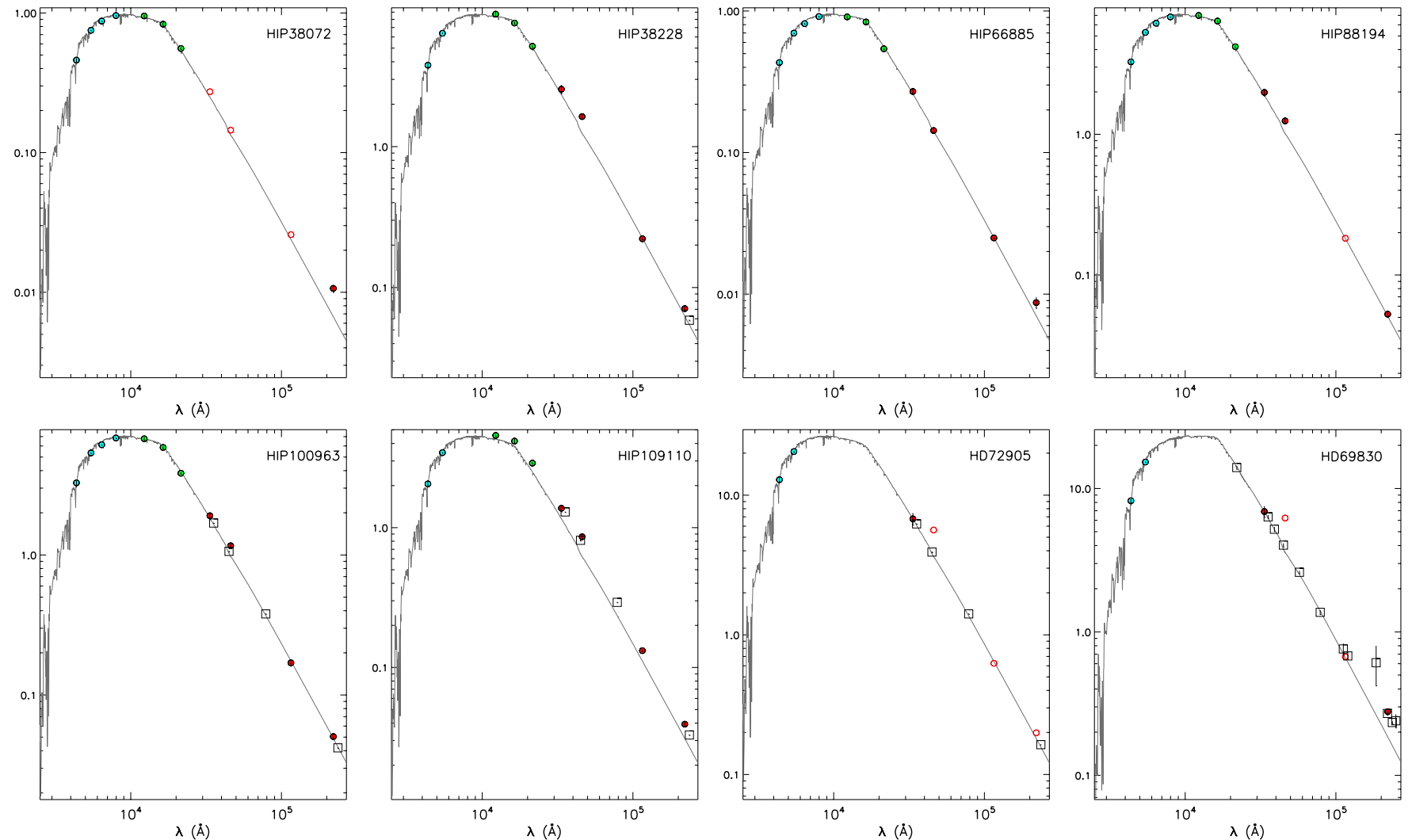

Figure 3. Observed fluxes vs. photospheric models (from Castelli \& Kurucz 2004) for stars still having anomalous WISE colors after imposing more stringent requirements on the source extraction. Fluxes, $F_{v}(\mathrm{Jy})$, are derived from optical (cyan), 2MASS (green), and WISE (red) magnitudes as described in the text: filled (open) symbols identify WISE photometric measurements that do (not) satisfy the requirements discussed in Section 4, including the stricter $\chi^{2}$ extraction threshold. Open squares are independent flux measurements available in the literature (Carpenter et al. 2008; Plavchan et al. 2009; Beichman et al. 2011).

(A color version of this figure is available in the online journal.)

class of object is thought to be very rare compared to most of the known cold Kuiper-belt-type disks, especially around old main-sequence stars (e.g., Bryden et al. 2006; Wyatt 2008). In Figure 1, it is interesting to include for comparison HD 69830 (HIP 40693), a $\sim 2-4$ Gyr old, solar metallicity dwarf known to host a warm disk closer than $\sim 1 \mathrm{AU}$ (Beichman et al. 2005), as well as three Neptune-mass planets within 0.6 AU (Lovis et al. 2006). Although HD 69830 has a $T_{\text {eff }}$ somewhat cooler than the bulk of our stars (Sousa et al. 2008), it shows a clear excess in $W 2$ and $W 4$, while $W 1$ and $W 3$ are barely affected. A somewhat similar trend is also shown by HD 72905 (HIP 42438), a $\sim 0.2$ Gyr old, solar-like star surrounded by hot dust (Beichman et al. 2006). ${ }^{11}$

However, producing emission in $W 2$ while keeping the other two contiguous bands essentially unaffected requires major finetunings, thus also rendering the disk interpretation difficult. At first, interpreting all anomalous WISE colors as suspected and/or poor photometry seems difficult because of the various quality constraints imposed (Section 2), although a number of W2 excesses have a saturated pixel fraction higher than usual (see Section 2) and more stringent cuts do alleviate the problem. Kennedy \& Wyatt (2012) have conducted a thorough study of stars in the Kepler field using WISE photometry to identify

\footnotetext{
11 HD 69830 satisfies all photometric quality requirements listed in Section 2, apart from missing a 2MASS counterpart within 3 arcsec. Note though that this star has a proper motion of $\sim 1 \operatorname{arcsec} \mathrm{yr}^{-1}$. HD 72905 was in our initial sample of Section 2, but was discarded because ext $=1$. This flag implies that the profile fit of the photometry is not optimal (namely, $\chi^{2}=3.6$ in $W 3$ ), although it is still not associated with any 2MASS Extended Source and all other photometric quality requirements of Section 2 are satisfied.
}

disk candidates and concluded in fact that spurious detections are less likely when using photometric measurements with source extraction $\chi^{2}$ smaller than $2,1.5,1.2$, and 1.2 in $W 1-4$, respectively. The requirement ext $=0$ we adopt is in fact less stringent (Section 2); the tighter $\chi^{2}$ constraints listed above are satisfied by $98 \%(W 1), 80 \%(W 2), 53 \%(W 3)$, and $93 \%(W 4)$ of the stars used in Section 3 and by 100\% (W1), 50\% (W2), 50\% $(W 3)$, and $95 \%(W 4)$ of the stars in Table 4. This suggests that any excess we see in $W 4$ might be real, but it casts some doubts on $W 2$. Adopting these constraints, the number of outliers in Table 4 is considerably reduced, although a number of them still remain: HIP 109110 and objects with excess in $W 2$ only (HIP 38228, HIP 88194, HIP 100963) and W4 only (HIP 38072 and HIP 66885).

For these six stars (plus HD 69830 and HD 72905), photospheric models tailored to the measured spectroscopic parameters are compared to fluxes derived from the adopted photometry (Figure 3). The absolute calibration of those model fluxes is done by forcing them to return synthetic $V$ magnitudes that match the observed ones. We also tested on the full sample of stars in Section 2 that the mean difference in physical fluxes is $0.25 \%$ and never exceeds $1.5 \%$, if instead doing the absolute calibration using angular diameters obtained from the IRFM. These differences are essentially indistinguishable on the scale of the plot and are taken into account when computing the flux uncertainty associated with each photometric measurement. Magnitudes in the $B V(R I)_{\mathrm{C}} J H K_{s}$ system are converted into fluxes using the same absolute calibration adopted in Casagrande et al. (2010), which has an intrinsic uncertainty of the order of $1 \%-2 \%$. 
Because of the aforementioned difference between using $V$ magnitudes and angular diameters, we have increased the global flux error to a conservative $3 \%$. Similarly, for the WISE system, we have adopted the absolute calibration and errors from Jarrett et al. (2011), further increasing the latter by $1 \%$, which also takes into account the possible zero-point offset discussed in the Appendix. Observed magnitude errors are then added to the aforementioned uncertainties regarding the absolute flux of each band. As already expected from color indices, any difference with respect to photospheric models in $W 2$ and $W 4$ is significant, and it does not stem from uncertainties on the flux scale or in the observed magnitudes. The advantage of fitting photospheric models instead of using color indices is that we are now able to better quantify the observed anomalies. The same comparison with synthetic spectra has also been done for all other stars in our sample not showing anomalous WISE colors, and indeed there are no mismatches between synthetic spectra and observations, thus validating the overall flux scale we adopt and also excluding major model deficiencies in those bands for solar-like stars (but see Kennedy \& Wyatt 2012, for possible model inaccuracies at cooler $T_{\text {eff }}$ ).

As we have already discussed, adding a cool companion does not modify the energy distribution in a way that is able to explain the observations, apart from HIP 109110, which longward of the $J$ band shows fluxes systematically higher than predicted. This likely indicates the presence of a cool companion (which we are able to fit with a model having $T_{\text {eff }} \sim 4000 \mathrm{~K}$ ), an interpretation that is consistent with its suspected binarity (Frankowski et al. 2007) and with the linear trend observed in its radial velocity (Nidever et al. 2002). This infrared excess is then further confirmed by the Spitzer-IRAC and MIPS measurements (Carpenter et al. 2008), shown in Figure 3 as open squares.

The W2 measurements for HD 69830 and HD 72905 do not pass the more stringent requirements we impose on the source extraction. The spurious nature of $W 2$ photometry for these two stars is then confirmed by the absence of excess in other measurements at similar wavelengths (Carpenter et al. 2008; Beichman et al. 2011). Note, though, that for HD 69830, the Infrared Spectrograph, Spitzer-MIPS, and IRAS data (Beichman et al. 2011) confirm the excess we see in $W 4$. Thus, we are inclined to regard any $W 2$ excess among our stars as artificial, even if the source extraction is fine; this is further supported by the fact that in Figure 3 the W2 photometry of HIP 100963 does not agree with Spitzer-IRAC (Carpenter et al. 2008). No additional measurements around $4.6 \mu$ m exist for HIP 38228 and HIP 88194, but from the previous discussion, and because these excesses seem rather challenging to interpret when contiguous bands agree well with photospheric models, we also conclude that their nature is likely spurious.

Finally, for HIP 38072 and HIP 66885, the deviation from photospheric models only starts in $W 4$. Despite these two stars being the faintest among those in Table 4, so far our adopted quality constraints have been enough to discard unreliable $W 4$ measurements, and what we see could indeed be the signature of debris disks around these two stars. For HIP 38072, we have a measured flux of $10.7 \pm 0.7 \mathrm{mJy}$ and a photospheric prediction of $6.7 \pm 0.2 \mathrm{mJy}$, thus resulting in an excess ratio of 1.6 with a $5 \sigma$ significance, while for HIP 66885 the measured flux is $8.7 \pm 0.8 \mathrm{mJy}$ versus a photospheric prediction of $7.0 \pm 0.2 \mathrm{mJy}$, the excess ratio being 1.2 at $2 \sigma$. Using the absence of emission in $W 3$ to constrain their temperature, we are able to easily fit these excesses with a blackbody, but measurements at other wavelengths are clearly required to confirm or discard the presence of any disk. Should these two detections be confirmed, and using the variance of the binomial distribution to derive a realistic error bar for such a low number statistic (e.g., Bevington 1969), the occurrence rate of debris disks at $22 \mu \mathrm{m}$ from our solar-like sample would thus be $3 \% \pm 2 \%$, in good agreement with $\sim 4 \%$ estimated by Trilling et al. (2008) at $24 \mu \mathrm{m}$.

\section{CONCLUSIONS}

The uncertainty on the zero point of the effective temperature scale has been addressed using one of the most stringent photometric constraints available, i.e., the infrared colors of the Sun, determined here in a model-independent way via LDRs. Such an analysis leads to an excellent agreement with the indices derived using the $T_{\text {eff }}$ scale of Casagrande et al. (2010), thus confirming its accuracy. Effective temperatures have also been determined by implementing the IRFM in the WISE system, thus validating the overall consistency between the 2MASS and WISE absolute calibration. This work also shows the importance of using solar twins for the absolute calibration of photometric quantities, something that is becoming increasingly more important in the era of large all-sky photometric surveys.

However, while 2MASS magnitudes are very well suited for the purpose of determining $T_{\text {eff }}$ - once stars affected by binarity are removed and/or using the full quality and flag information available in 2MASS to discard bad photometry - this does not necessarily hold for the WISE data. Special attention to WISE's photometric quality flags and source extraction information must be paid, yet a number of excess emissions in $W 2$ seem artificial. In this respect, 2 MASS magnitudes lie in a "sweet spot," enough in the red to sample the Planck tail, yet largely unaffected by contamination and/or flux excess, either real or spurious.

At this stage, it is still unclear whether all WISE midinfrared anomalies found stem from bad measurements or are instead associated with real physical phenomena. As either case is possible, those stars are clearly not representative of the Sun and have not been used to derive its colors. It is extremely difficult to interpret in a consistent manner objects showing intense excess in $W 2$ only (and in fact, comparison with independent measurements confirms the spurious nature), while on the contrary it seems genuine for $W 4$. The latter excess could be the signature of warm/hot debris disks, the best candidates from our sample being HIP 38072 and HIP 66885. Data at longer wavelengths are clearly needed: should HIP 38072 be confirmed, it would be the first solar analog/twin $\left(T_{\mathrm{eff}}^{\mathrm{spec}}=5839 \mathrm{~K}, \log g=4.53 \mathrm{dex}\right.$, and $\left.[\mathrm{Fe} / \mathrm{H}]=0.06 \mathrm{dex}\right)$ found to host such a debris disk. This star is also relatively young $(2.4 \mathrm{Gyr})$ and has $\sim 4$ times more lithium than the Sun (Baumann et al. 2010), and it is included in our HARPS radial velocity monitoring, thus making it a potentially interesting target to gauge new insights into the planet-disk interaction.

We thank an anonymous referee for a prompt report. I.R.'s work was performed under contract with the California Institute of Technology (Caltech) funded by NASA through the Sagan Fellowship Program. J.M. acknowledges support from FAPESP (2010/17510-3) and CNPq. This publication makes use of data products from the Two Micron All Sky Survey, which is a joint project of the University of Massachusetts and the Infrared Processing and Analysis Center/California Institute of Technology, funded by the National Aeronautics and Space Administration and the National Science Foundation. This publication makes use of data products from the Wide-field 

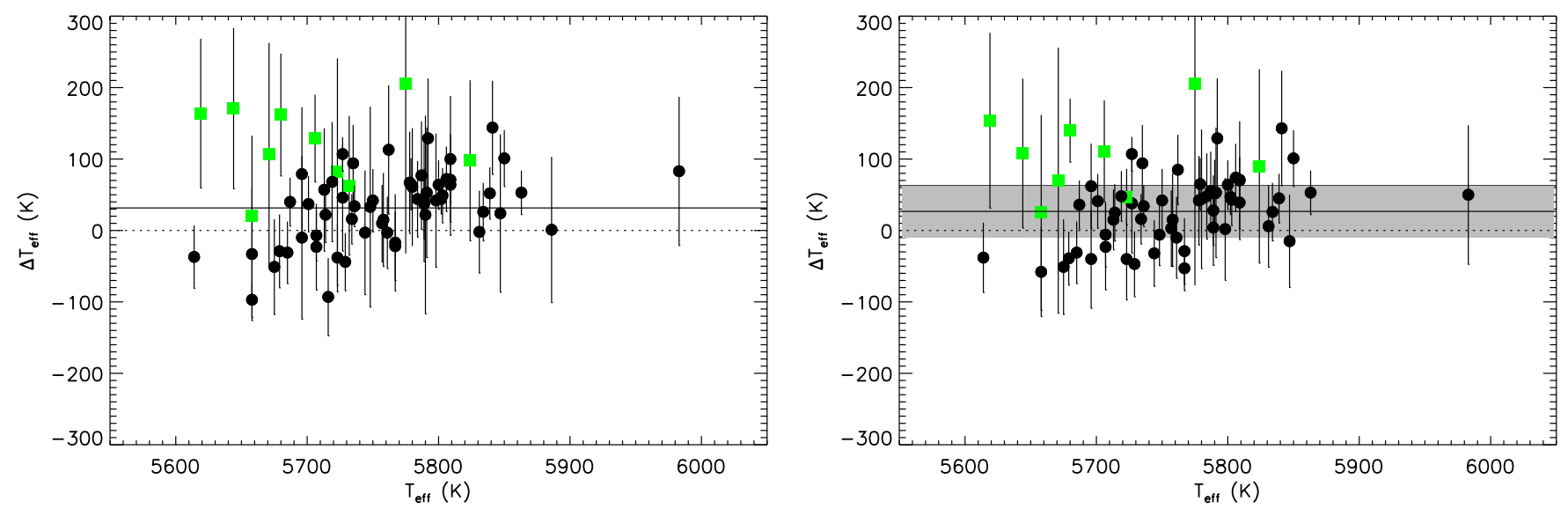

Figure 4. $\Delta T_{\text {eff }}(2 \mathrm{MASS}-$ WISE) when implementing one or another system in the IRFM. Filled squares are stars having $V-W 2$ and $V-W 4$ redder than the thresholds discussed in Section 2 and are not used to derive the mean difference (continuous line). Left panel: using in the IRFM at least two bands among $W 1-4$. Right panel: when restricting to at least two bands among W1-3. The gray shaded area is the systematic offset ( $\pm 36 \mathrm{~K})$ allowed by the uncertainty in WISE's absolute calibration.

(A color version of this figure is available in the online journal.)

Infrared Survey Explorer, which is a joint project of the University of California, Los Angeles, and the Jet Propulsion Laboratory/California Institute of Technology, funded by the National Aeronautics and Space Administration.

\section{APPENDIX}

\section{THE INFRARED FLUX METHOD USING WISE PHOTOMETRY}

The availability of 2MASS and WISE photometry for most of the stars in our sample permits us to run the IRFM using these two systems separately and to check that consistent effective temperatures are derived. The implementation of the method is identical to that described in Casagrande et al. (2006, 2010), i.e., for each star, the bolometric flux is recovered using all available broadband optical/near-infrared/mid-infrared colors, while infrared fluxes needed to derive $T_{\text {eff }}$ are now computed using 2MASS or WISE photometry, respectively.

For solar-type stars, the main driver in setting the zero point of the $T_{\text {eff }}$ scale is the absolute calibration of infrared bands. We have already discussed and tested that of 2MASS (Casagrande et al. 2010 and references therein), thus focusing on the WISE system here. We adopt the $W 1-4$ relative system response curves ${ }^{12}$ and physical monochromatic fluxes $F_{\lambda}$ (iso) from Jarrett et al. (2011). The latter are built on the same absolute basis established for the Spitzer Space Telescope, ultimately constructed on the "Cohen-Walker-Witterborn" framework (Cohen et al. 2003 and references therein) and tied directly to the absolute mid-infrared calibrations by the Midcourse Space Experiment (Price et al. 2004). These WISE fluxes have an expected overall systematic uncertainty of the order of $\sim 1.5 \%$ and define the Vega zero-magnitude attributes upon which the effective temperatures we derive in this system directly depend. For each star, we used the photometric constraints discussed in Section 2 and derived $T_{\text {eff }}$ if at least two WISE bands were simultaneously available. $W 4$ magnitudes have the largest photometric errors (Section 2), thus showing the weakest correlation with $T_{\text {eff }}$; indeed, the scatter in the comparison with the effective temperatures obtained using 2MASS magnitudes reduces when $W 4$ is not implemented in the IRFM (Figure 4).

\footnotetext{
$\overline{12}$ http://wise2.ipac.caltech.edu/docs/release/allsky/expsup/sec4_4h.html
}

The maximum zero-point uncertainty in the effective temperatures derived using WISE photometry can easily be estimated by increasing or decreasing the adopted absolute calibration according to the error reported in Jarrett et al. (2011). As expected, for the stellar parameter space covered here, the effect is essentially a constant offset of $\pm 36 \pm 0.4 \mathrm{~K}(\sigma=3 \mathrm{~K})$. Note that our reference 2MASS effective temperatures also have a zero-point uncertainty of the order of $20 \mathrm{~K}$ (Casagrande et al. 2010). The 25-30 K mean difference when using 2MASS or WISE magnitudes in the IRFM is thus fully consistent within the uncertainties, and it would disappear if the WISE absolute calibration had been decreased by about $1 \%$ (or conversely, the 2MASS absolute calibration increased by the same amount). Such a good agreement is not entirely unexpected, since the 2MASS and the WISE absolute calibration are built within the same "Cohen-Walker-Witterborn" framework: nevertheless, the fact that the 2MASS absolute calibration has been independently verified using solar twins confirms that the accuracy of the infrared absolute calibration also extends to the mid-infrared regime probed by WISE.

Note added in proof. The reason for the anomalously bright W2 magnitudes likely stems from problems in the profile fitting photometry for bright saturated stars, see http://wise2.ipac.caltech. edu/docs/release/allsky/expsup/sec6_3c.html

\section{REFERENCES}

Alonso, A., Arribas, S., \& Martinez-Roger, C. 1996, A\&A, 313, 873 Baumann, P., Ramírez, I., Meléndez, J., Asplund, M., \& Lind, K. 2010, A\&A, 519, A87

Beichman, C. A., Bryden, G., Gautier, T. N., et al. 2005, ApJ, 626, 1061 Beichman, C. A., Lisse, C. M., Tanner, A. M., et al. 2011, ApJ, 743, 85 Beichman, C. A., Tanner, A., Bryden, G., et al. 2006, ApJ, 639, 1166 Bessell, M. S., Castelli, F., \& Plez, B. 1998, A\&A, 333, 231

Bevington, P. R. 1969, Data Reduction and Error Analysis for the Physical Sciences (New York: McGraw-Hill)

Biazzo, K., Frasca, A., Catalano, S., \& Marilli, E. 2007, Astron. Nachr., 328, 938

Bilir, S., Ak, S., Karaali, S., et al. 2008, MNRAS, 384, 1178

Bilir, S., Karaali, S., Ak, S., et al. 2011, MNRAS, 417, 2230

Boyajian, T. S., McAlister, H. A., van Belle, G., et al. 2012a, ApJ, 746, 101

Boyajian, T. S., von Braun, K., van Belle, G., et al. 2012b, ApJ, 757, 112

Bryden, G., Beichman, C. A., Trilling, D. E., et al. 2006, ApJ, 636, 1098

Burrows, A., Sudarsky, D., \& Hubeny, I. 2006, ApJ, 640, 1063

Campins, H., Rieke, G. H., \& Lebofsky, M. J. 1985, AJ, 90, 896 
Carpenter, J. M., Bouwman, J., Silverstone, M. D., et al. 2008, ApJS, 179, 423

Casagrande, L., Portinari, L., \& Flynn, C. 2006, MNRAS, 373, 13

Casagrande, L., Ramírez, I., Meléndez, J., Bessell, M., \& Asplund, M. 2010, A\&A, 512, A54

Casagrande, L., Schönrich, R., Asplund, M., et al. 2011, A\&A, 530, A138

Castelli, F., \& Kurucz, R. L. 2004, arXiv:astro-ph/0405087

Cayrel de Strobel, G. 1996, A\&AR, 7, 243

Cohen, M., Megeath, S. T., Hammersley, P. L., Martín-Luis, F., \& Stauffer, J. 2003, AJ, 125, 2645

Cousins, A. W. J. 1987a, Mon. Notes Astron. Soc. South Afr., 46, 144

Cousins, A. W. J. 1987b, Observatory, 107, 80

Cutri, R. M., Skrutskie, M. F., van Dyk, S., et al. 2003, VizieR Online Data Catalog, 2246, 0

Cutri, R. M., Wright, E. L., Conrow, T., et al. 2012, Explanatory Supplement to the WISE All-Sky Data Release Products, Technical Report, 1

Datson, J., Flynn, C., \& Portinari, L. 2012, MNRAS, 426, 484

Frankowski, A., Jancart, S., \& Jorissen, A. 2007, A\&A, 464, 377 Gray, D. F. 1994, PASP, 106, 1248

Gustafsson, B., Edvardsson, B., Eriksson, K., et al. 2008, A\&A, 486, 951

Holmberg, J., Flynn, C., \& Portinari, L. 2006, MNRAS, 367, 449

Huber, D., Ireland, M. J., Bedding, T. R., et al. 2012, ApJ, 760, 32

Jarrett, T. H., Cohen, M., Masci, F., et al. 2011, ApJ, 735, 112

Johnson, H. L. 1965, Commun. Lunar Planet. Lab., 3, 73

Kennedy, G. M., \& Wyatt, M. C. 2012, MNRAS, 426, 91
Kirkpatrick, J. D., Cushing, M. C., Gelino, C. R., et al. 2011, ApJS, 197, 19 Kovtyukh, V. V., Soubiran, C., Belik, S. I., \& Gorlova, N. I. 2003, A\&A, 411, 559

Lovis, C., Mayor, M., Pepe, F., et al. 2006, Nature, 441, 305

Mainzer, A., Cushing, M. C., Skrutskie, M., et al. 2011, ApJ, 726, 30

Meléndez, J., Asplund, M., Gustafsson, B., \& Yong, D. 2009, ApJ, 704, L66

Meléndez, J., Dodds-Eden, K., \& Robles, J. A. 2006, ApJ, 641, L133

Meléndez, J., \& Ramírez, I. 2007, ApJ, 669, L89

Meléndez, J., Schuster, W. J., Silva, J. S., et al. 2010, A\&A, 522, A98

Nidever, D. L., Marcy, G. W., Butler, R. P., Fischer, D. A., \& Vogt, S. S. 2002, ApJS, 141, 503

Nordström, B., Mayor, M., Andersen, J., et al. 2004, A\&A, 418, 989

Plavchan, P., Werner, M. W., Chen, C. H., et al. 2009, ApJ, 698, 1068

Price, S. D., Paxson, C., Engelke, C., \& Murdock, T. L. 2004, AJ, 128, 889

Ramírez, I., \& Meléndez, J. 2005, ApJ, 626, 465

Ramírez, I., Meléndez, J., \& Asplund, M. 2009, A\&A, 508, L17

Ramírez, I., Michel, R., Sefako, R., et al. 2012, ApJ, 752, 5

Ridgway, S. T., Joyce, R. R., White, N. M., \& Wing, R. F. 1980, ApJ, 235, 126

Rieke, G. H., Blaylock, M., Decin, L., et al. 2008, AJ, 135, 2245

Sousa, S. G., Santos, N. C., Mayor, M., et al. 2008, A\&A, 487, 373

Stebbins, J., \& Kron, G. E. 1957, ApJ, 126, 266

Trilling, D. E., Bryden, G., Beichman, C. A., et al. 2008, ApJ, 674, 1086

Wamsteker, W. 1981, A\&A, 97, 329

Wright, E. L., Eisenhardt, P. R. M., Mainzer, A. K., et al. 2010, AJ, 140, 1868

Wyatt, M. C. 2008, ARA\&A, 46, 339 